\title{
Multifractal analysis of growing surfaces
}

\author{
Ajay Chaudhari, Ching-Cher Sanders Yan, Shyi-Long Lee* \\ Department of Chemistry and Biochemistry, National Chung-Cheng University, Ming-Hsiung, Chia-Yi 621, Taiwan
}

Available online 27 August 2004

\begin{abstract}
Multifractal scaling analysis is applied to the growing surfaces of random deposition model. The effect of number of deposited particles and lattice size on multifractal spectra is studied. Three cases of the growing surfaces are considered: (1) Same total number of particles deposited on different square lattice so that the number of particles deposited per surface site is different. (2) Different total number of particles deposited on different square lattice so that the number of particles deposited per surface site is the same. (3) Different total number of particles deposited on same square lattice to study the effect of number of deposited particles on multifractal spectra. The multifractal spectra are related to the surface irregularity of the growing surfaces. It has been observed that the surface with more surface roughness gives greater non-linearity in $q-\tau(q)$ multifractal spectra results in wider range of $\alpha$ values in $\alpha-f(\alpha)$ multifractal spectra.
\end{abstract}

(C) 2004 Elsevier B.V. All rights reserved.

PACS: 68.55.-a; 68.35.Bs; 61.43.Hv; 81.15.-z

Keywords: Growing surface; Multifractal analysis; Surface roughness

\section{Introduction}

Study of growing surfaces and of their physical and structural properties is consequently of great interest for many practical purposes [1-3]. Growth by vapor deposition is an effective process for producing high quality materials. During the growth, the initially flat surface becomes rough. On the microscopic level, growth is usually investigated using discrete growth models. Random deposition model is one of them. The parameters associated with growing surface process affect the development of the interfaces in varying degree.

\footnotetext{
* Corresponding author.

E-mail address: chesll@ccu.edu.tw (S.-L. Lee).
}

There are various studies on growing surfaces [4-8]. Amitrano et al. [4] have used Green's function technique to calculate growth probability distribution in kinetic aggregation process and obtained multifractal plots. Experimental evidence of multifractality for diffusion-limited aggregation (DLA) surface is given by Nittmann et al. [5]. The multifractality concept is applied to self-affine fractal by Barabasi and Vicsek [6]. Hou et al. [7] have prepared thin film of $\mathrm{C}_{60} / \mathrm{Ag}$ by deposition method and applied multifractal analysis to the surface of the film. Family has applied dynamic scaling theory to the growing surface of random deposition model [8]. In this study, we have applied multifractal scaling analysis to the growing surfaces of random deposition model $[1,8]$. We have studied in particular how the parameters, lattice size and number of depositing particle, affect the growing surfaces 
through multifractal scaling analysis. The present paper is organized as follows. In Section 2, the method of generation of rough surfaces is given, Section 3 gives the details of the multifractal scaling analysis. In Section 4, we present and discuss the results. Conclusions are given in Section 5.

\section{Model}

The random deposition model [8] has been used for generating the rough surfaces. It is the simplest deposition model. In random deposition model, particles simply rain down onto a smooth surface. Particles move along straight-line trajectories until they reach the top of the column in which they are dropped, at which point they stick to the deposit and become part of the aggregate. As there is no horizontal correlation between neighboring columns, the surface is extremely rough.

Following three cases of growing surfaces are considered and studied how multifractal spectra changes for these three cases.

Case I. Same total number of particles deposited on different square lattice so that the number of particles deposited per surface site is different. The $3 \times 10^{5}$ particles are deposited on a square lattice of linear dimension $L=100,150,200$ and 250 lattice units.

Case II. Different total number of particles deposited on different square lattice so that the number of particles deposited per surface site is the same. The $10^{5}, 225000,4 \times 10^{5}$ and 625000 particles are deposited on a square lattice of linear dimension $L=100,150,200$ and 250 lattice units, respectively.

Case III. Different total number of particles deposited on same square lattice to study the effect of number of deposited particles on multifractal spectra. The $10^{5}, 2 \times 10^{5}, 3 \times 10^{5}$ and $4 \times 10^{5}$ particles are deposited on square lattice of linear dimension $L=100$ lattice units.

The surface roughness is obtained as

$R=\left[\frac{\sum_{i}\left(h_{i}-H\right)^{2}}{N_{\mathrm{s}}}\right]^{1 / 2}$

where $h_{i}$ is the height of the $i$ th column, $H=\sum_{i} h_{i} / N_{\mathrm{s}}$ the mean deposition height and $N_{\mathrm{s}}$ the number of surface sites.
After generating the rough surface, the growth probability of each surface site is recorded. We have applied the multifractal scaling which relates the analysis of the distribution of the growth probabilities over the length of the rough surface. The steps in the multifractal scaling analysis are given in Section 3. For more details one can refer to the article by Halsey et al. [9]. The results presented here are the average of 25 runs.

\section{Multifractal analysis}

The three basic equations in the multifractal scaling analysis are

$$
\begin{aligned}
& M_{q} \sum_{i} P_{i}^{q}=\sum_{p} n(P) P^{q} \propto L^{-\tau(q)} \\
& \tau(q)=q \alpha(q)-f(\alpha) \\
& \frac{\mathrm{d}}{\mathrm{d} q}[\tau(q)]=\alpha(q)
\end{aligned}
$$

where $M_{q}$ is the $q$ th-order moment of growth probability distribution, $\tau(q)$ the scaling exponent, $P_{i}$ the growth probability of site $i, n(P)$ the number of sites with growth probability $P$ and $L$ the length of the surface. The exponents $\alpha$ and $f(\alpha)$ are the singularity exponents and fractal dimension, respectively. Among these three equations, Eq. (3) is obtained by the following scaling assumptions used in the limiting case of large $L$

$P(q) \propto L^{-\alpha(q)}$

$n[P(q)] \propto L^{f(\alpha)}$

where $P(q)$ denotes the value of $P$ that dominates the sum in Eq. (2) for the qth-order moment. Substitution of Eqs. (5) and (6) into Eq. (2) and taking the logarithm of the maximum elements as an approximation of the logarithm of the sum yields Eq. (3). Finally, Eq. (4) represents the value of $\alpha$ that dominates the summation term in Eq. (2) and it can be transferred into a more explicit form,

$\alpha(q)=\frac{\mathrm{d}}{\mathrm{d} q}[\tau(q)]=\frac{\sum P_{i}^{q} \ln \left(P_{i}\right)}{\sum P_{i}^{q} \ln (1 / L)}$ 
In practice, the quantities $\tau(q), \alpha(q)$ and $f(\alpha)$ can be computed as follows. The growth probability distribution is first determined from the simulation. Then for each $L, \tau(q)$ is calculated from Eq. (2). $\alpha(q)$ is then computed from Eq. (7). By knowing $\tau$ and $\alpha$, one can compute $f(\alpha)$ from Eq. (3). The multifractal analysis gives two curves: (1) $q$ versus $\tau(q)$ and (2) $\alpha$ versus $f(\alpha)$.

\section{Results and discussion}

From simulation, for each case, the growth probability of different surface sites is obtained first as $P_{i}=$ $h_{i} / N_{\text {total }}$, where $h_{i}$ is the height of $i$ th column and $N_{\text {total }}$ the total number of particles deposited.

For case I, $3 \times 10^{5}$ particles are deposited on square lattice of linear dimension $L=100,150,200$ and 250 lattice units so that the number of particles per surface site is different for different $L$. The surface roughness for these four surfaces calculated using Eq. (1) is 5.51, $2.72,1.82$ and 1.37 , respectively. It indicates that the surface for $L=100$ is the roughest and that for $L=250$ is the smoothest among the four surfaces. The multifractal spectra for these four surfaces are shown in Fig. 1a. The non-linearity in $q-\tau(q)$ indicates multifractality. The difference in non-linearity for the four surfaces can clearly be seen from Fig. 1a. Since the surface for $L=100$ is the roughest among the four, the $q-\tau(q)$ curve is more deviated from linearity for this surface than the others. The curves coincide neither in low nor in high values of $q$ indicates that the number of surface sites with low growth probability (for low values of $q$ ) and with high growth probability (for high values of $q$ ) are quite different for the four surfaces. As the surface roughness decreases, the non-linearity in the $q-\tau(q)$ curve decreases representing a homogeneous growth probability distribution.

The greater non-linearity for $L=100$ results in wider range of $\alpha$ values in Fig. 1a. The width of $\alpha-$ $f(\alpha)$ spectra also reflects the difference in four surfaces. The roughest surface has widest $\alpha-f(\alpha)$ spectra. The range of $\alpha$ values also represents the range of height distribution of probabilities. More the rough surface, wider is the distribution of the growth probability. The range of $\alpha$ values decreases with decrease in surface roughness indicates that the height distribution is more uniform for the smoother surface than that or rougher surface.

The multifractal spectra are used not only to characterize the surface roughness but it can also be used for characterizing the shape of peaks and valleys between different rough surfaces. As can be seen from Fig. 1a, $f(\alpha)$ figures are asymmetric with respect to $\alpha$ for all the four surfaces. The difference in the $f(\alpha)$ spectrum for these four surfaces can also be seen at the low and high $\alpha$ values. As the surface roughness decreases, the right extreme $f(\alpha)$ value becomes larger and left are smaller. This implies that as the surface roughness decreases, the low growth probability part is associated with larger sets but the higher growth probability part is connected to very small sets and as a result it produces a relatively homogeneous growth probability distribution pattern.

For case II, different total number of particles deposited on different square lattice so that number of particles deposited per surface site is the same. The surface roughness for the four surfaces of $L=100$, 150,200 and 250 is $3.20,3.17,3.16$ and 3.15 , respectively. It can be seen that even though there are same number of particles deposited per surface site, the surface roughness is slightly different for these four surfaces. The multifractal spectra for these four surfaces are shown in Fig. 1b. The $q-\tau(q)$ curves are non-linear for these four surfaces and these curves coincide in positive $q$ region whereas these are slightly separated in negative $q$ region. The deviation from linearity is highest for $L=100$ and lowest for $L=250$. This indicates that even though same number of particles deposited per surface site, the number of surface sites with lowest growth probability, which dominates the sum in Eq. (2) is slightly different for different $L$. The curves coincide in positive region indicates that the number of sites with high growth probability is nearly equal for these four surfaces. The range of $\alpha$ values is also slightly different for these surfaces and it decreases with increasing $L$. Here also the $f(\alpha)$ curves shift upward for high $\alpha$ values with increasing $L$ whereas for low $\alpha$ values, these are almost identical.

In Fig. 1c, effect of number of deposited particles on multifractal spectra is shown. The $10^{5}, 2 \times 10^{5}, 3 \times$ $10^{5}$ and $4 \times 10^{5}$ particles are deposited on same square lattice of $L=100$ lattice units and the surface roughness obtained is $3.20,4.51,5.51$ and 6.34 , respectively. 
(a)
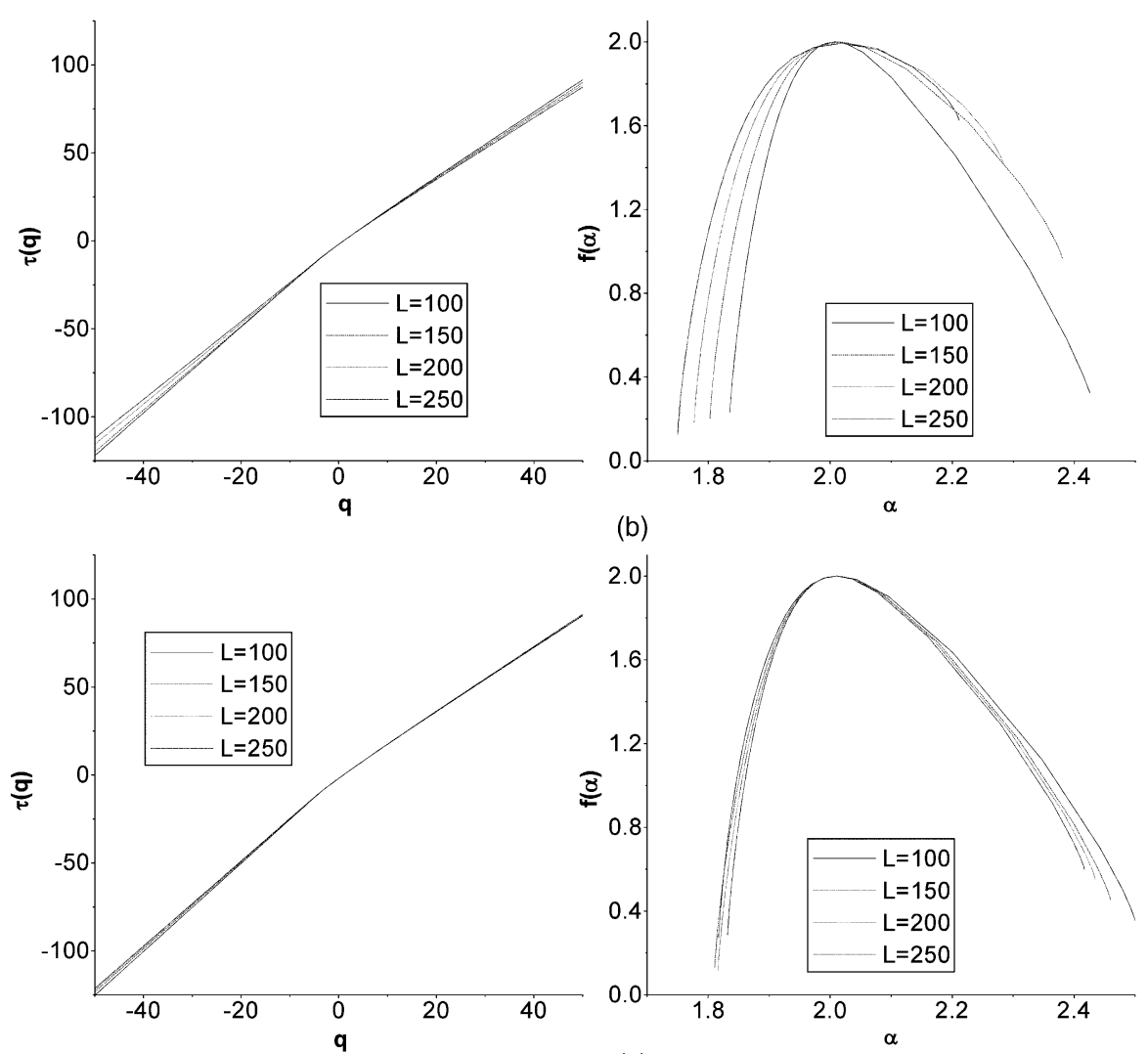

(b)
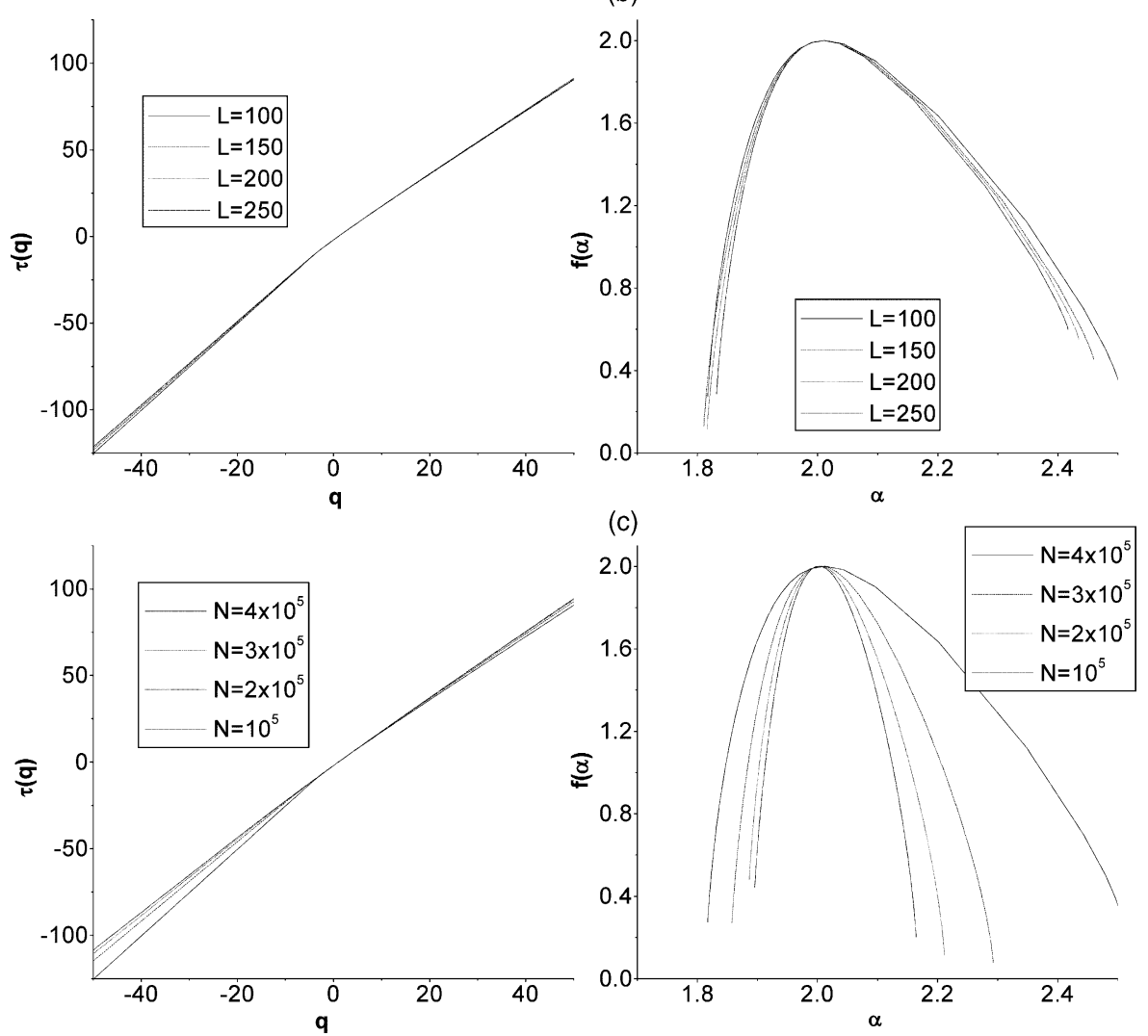

Fig. 1. Multifractal spectra for: (a) same number of particles deposited per site; (b) different number of particles deposited per site; (c) different total number of particles deposited on the same square lattice.

It can be seen that the number of deposited particles also affects the multifractal spectra (i.e. surface roughness). Higher the number of deposited particles, more is the surface roughness and greater is the deviation from linearity in $q-\tau(q)$ plot results in wider range of $\alpha$ values in $\alpha-f(\alpha)$ multifractal plot. As the number of deposited particles increases the range of $\alpha$ values also increases.

From the above study it can be concluded that the surface with higher surface roughness has greater non- 
linearity in $q-\tau(q)$ curves and gives wider range of $\alpha$ values in $\alpha-f(\alpha)$ multifractal plot. Our results agree well with the experimental results of Hou et al. [7]. Hou et al. have prepared thin film of $\mathrm{C}_{60} / \mathrm{Ag}$ by codepositing $\mathrm{C}_{60}$ and $\mathrm{Ag}$ on $\mathrm{NaCl}$ substrate and also observed that the surface with more roughness gives wider range of $\alpha$ values in multifractal plot.

\section{Conclusions}

We have studied the growing surfaces by random deposition model. The multifractal spectra are obtained by using the growth probability of different surface sites. The surface roughness is related to the multifractal parameters. The width of the multifractal spectra increases with an increase in surface roughness. In addition to surface roughness, the multifractal spectra also provided the information about the number of maximum and minimum growth probability sites.

\section{Acknowledgements}

The financial support from National Science Council, Taiwan is gratefully acknowledged.

\section{References}

[1] F. Family, T. Vicsek, Dynamics of Fractal Surfaces, World Scientific, Singapore, 1991.

[2] A. Bunde, S. Havlin, Fractals and Disordered Systems, Springer-Verlag, New York, 1991.

[3] T. Vicsek, Fractal Growth Phenomena, World Scientific, Singapore, 1989.

[4] C. Amitrano, A. Coniglio, F. di Liberto, Phys. Rev. Lett. 57 (1986) 1016.

[5] J. Nittmann, H.E. Stanley, E. Touboul, G. Daccord, Phys. Rev. Lett. 58 (1987) 619.

[6] A.L. Barabasi, T. Vicsek, Phys. Rev. A 44 (1991) 2730.

[7] J.G. Hou, W. Yan, X. Rui, et al., Phys. Rev. E 58 (1998) 2213.

[8] F. Family, J. Phys. A: Math. Gen. 19 (1986) L441.

[9] T.C. Halsey, M.H. Jensen, L.P. Kadanoff, O. Procaccia, B.I. Shraiman, Phys. Rev. A 33 (1986) 1141. 